Referencia para citar este artículo: Molina, M. F., Raimundi, M. J. \& Gimenez, M. (2017). Los posibles sí mismos de los adolescentes de Buenos Aires. Revista Latinoamericana de Ciencias Sociales, Niñez y Juventud, 15 (1), pp. 455-470.

\title{
Los posibles sí mismos de los adolescentes de Buenos Aires*
}

\author{
María FERNANDA Molina** \\ Becaria Postdoctoral del Consejo Nacional de Investigaciones Científicas y Técnicas (Conicet), Argentina. \\ MARÍA JULIA RAIMUNDI*** \\ Becaria Postdoctoral del Consejo Nacional de Investigaciones Científicas y Técnicas (Conicet), Argentina.
}

MARIEL GIMENEZ ${ }^{* * * *}$

Especialista en evaluación psicológica-Investigadora en formación UBACyT, Argentina.

\section{Artículo recibido en octubre 1 de 2015; artículo aceptado en febrero 9 de 2016 (Eds.)}

- Resumen (analítico): El objetivo de este trabajo fue explorar y describir los posibles sí mismos (PSs) de un grupo de adolescentes de la Ciudad de Buenos Aires (C.A.B.A.). Participaron de este estudio 72 adolescentes (69.4\% mujeres) de una escuela privada de la C.A.B.A. $(M=15.04 ; D E$ =1.56). Los adolescentes respondieron un cuestionario de preguntas abiertas en el que se les pidió que realicen un listado de sus PSs cuando terminen el secundario. Se realizó un análisis de contenido temático de las respuestas y un conteo de frecuencias de las categorías. Los adolescentes generaron PSs en cinco grandes áreas: personal, académico/ocupacional, física, social, y de satisfacción y logros. El área más prevalente es la académico/ocupacional. La mayoría de los adolescentes generaron PSs en una o dos áreas.

Palabras clave: autoconcepto, adolescencia, investigación cualitativa, adolescentes en Buenos Aires (PsychInfo Thesaurus).

\section{The possible selves of adolescents from Buenos Aires}

- Abstract (analytical): The aim of this study is to explore and describe the possible selves (PSs) of a group of teenagers from the City of Buenos Aires (C.A.B.A.). Participants were 72 adolescents $(69.4 \%$ female) from a private school in C.A.B.A. $(M=15.04 ; S D=1.56)$. Adolescents completed

\footnotetext{
* Este artículo de investigación científica y tecnológica (Área: Psicología / Sub-área: Psicología -incluye relaciones hombre-máquina) presenta resultados finales del proyecto de investigación "La visión del sí mismo en el futuro: Su relación con el autoconcepto actual y la percepción del funcionamiento familiar en la adolescencia”, financiado por el Consejo Nacional de Investigaciones Científicas y Técnicas (Res. № 4823/13, inició en abril de 2014 y finalizó en marzo de 2016) y por la Secretaría de Ciencia y Técnica de la Universidad de Buenos Aires (Proyecto UBACyT 20020130100434. Directora: Dra. Vanina Schmidt. Co-directora: Dra. Nora Leibovich de Figueroa). Estudio descriptivo-correlacional con diseño transversal.

Agradecimientos: Se agradece a los alumnos, docentes, y autoridades de la escuela en la que se realizó el estudio, su colaboración en este proyecto de investigación.

** Dra en Psicología. Becaria postdoctoral de Conicet. Filiación institucional: Conicet-Consejo Nacional de Investigaciones Científicas y Técnicas. UBA-Instituto de Investigaciones de la Facultad de Psicología de la Universidad de Buenos Aires. Correo electrónico: fernandamolina@psi.uba.ar

*** Dra en Psicología. Becaria postdoctoral de Conicet. Filiación institucional: Conicet-Consejo Nacional de Investigaciones Científicas y Técnicas. UBA-Instituto de Investigaciones de la Facultad de Psicología de la Universidad de Buenos Aires. Correo electrónico: jraimundi@psi.uba.ar

***** Lic en Psicología. Filiación institucional: UBA-Instituto de Investigaciones de la Facultad de Psicología de la Universidad de Buenos Aires. Correo electrónico: mariel.y.gimenez@gmail.com
} 
a questionnaire with open-ended questions in which adolescents were asked to make a list of their PSs when they finish high school. Thematic content analysis of responses and frequency counts of categories was conducted. Teens generated PSs in five major areas: academicloccupational, physical, social, and satisfaction and achievements. The most prevalent area is the academic/occupational. Most teenagers generated PSs in one or two areas.

Key words: Self-concept, adolescents, qualitative research, adolescents in Buenos Aires (PsychInfo Thesaurus).

\section{Os possíveis si mesmos de adolescentes de Buenos Aires}

- Resumo (analítico): O objetivo deste estudo foi explorar e descrever os possíveis si mesmos (PSS) de um grupo de adolescentes da Cidade de Buenos Aires (Caba). Este estudo envolveu 72 adolescentes (69.4\% mulheres) de uma escola particular da Caba $(M=15.04, D P=1.56)$. Os adolescentes responderam a um questionário de perguntas abertas, que foram convidados a fazer uma lista de seu PSs quando terminem o ensino médio. Análise de conteúdo temática das respostas e uma frequência de contagem das categorias foi realizada. Os adolescentes geraram PSs em cinco grandes áreas: acadêmica / profissional, físico, social, e pessoal de satisfação e realizações. A área mais prevalente é o acadêmico / profissional. A maioria dos adolescentes geraram PSs em uma ou duas áreas.

Palavras-chave: Auto-conceito, adolescência, pesquisa qualitativa, adolescentes em Buenos Aires (PsychInfo Thesaurus).

\section{-1. Introducción. -2. Método. -3. Resultados. -4. Discusión. -5. Conclusiones. -Lista de referencias.}

\section{Introducción}

La formación de la identidad es una de las tareas centrales que deben enfrentar los adolescentes. En esta etapa vital se crea el sí mismo en el que uno se podría convertir(Erikson, 1968) y el autoconcepto sufre importantes modificaciones (Harter \& Bukowski, 2012). En la adolescencia temprana, los jóvenes empiezan a usar descripciones más abstractas, pudiendo integrar diferentes perspectivas del sí mismo: cómo son, cómo fueron, cómo quieren ser, y cómo se presentan al mundo. De esta forma, la representación del sí mismo enfocada en el pasado y el futuro, evoluciona (Oyserman, 2001).

Es por esto que el estudio de los posibles sí mismos (PSs) es de particular importancia en esta etapa vital. Éstos constituyen el autoconocimiento que hace referencia al propio potencial y al futuro posible (Markus \& Nurius, 1986). Son los componentes multifacéticos del autoconcepto que se encuentran proyectados hacia el futuro (Oyserman \& Fryberg, 2006). Los PSs no son simples metas o aspiraciones. Es necesario que haya una representación de uno mismo siendo agente en esa situación, estando en dicho escenario, experimentándolo desde el interior (Erikson, 2007). Los PSs pueden ser más o menos realistas, pueden ser deseados o temidos, y percibidos como alcanzables o evitables (Markus \& Nurius, 1986, Oyserman \& Fryberg, 2006).

Para Markus y Nurius (1986) los PSs proveen un nexo entre el autoconcepto actual y la motivación ya que brindan la energía y los medios para alcanzar las metas, permiten desarrollar un plan, llevarlo adelante y supervisar su progreso por medio de la autoevaluación de competencias (Wurf \& Markus, 1991, citados en Shepard \& Marshall, 1999). Esto sucede principalmente cuando el contexto situacional es relevante para el PSs que se activa (Oyserman, Destin, \& Novin, 2015).

Por otra parte, la construcción de PSs funciona como un proceso cognitivo en el que 
se prueban imágenes futuras de uno mismo (Sica, 2009). Es por esto que son una expresión de la exploración de la identidad en tanto la construcción de dichas imágenes es una forma de explorar activamente quién uno podría ser (Cadely, Pittman, Kerpelman \& Adler-Baeder, 2011).

La proyección de sí mismos en el futuro tiene efectos en el bienestar(Oyserman \& James, 2011), la autoestima (Knox, Funk, Elliot \& Bush, 1998), los logros académicos, y el compromiso con la escuela y el estudio (Destin \& Oyserman, 2010) de los adolescentes. Asimismo, se vincula con problemas de conducta (Brewer, Gearing, Schwalbe \& Ibrahim, 2013), consumo de alcohol (Lee, Corte, Stein, Park, Finnegan \& Mccreary, 2015) y modera la relación entre el comportamiento negativo de los pares y el comportamiento violento de los adolescentes (Pierce, Schmidt \& Stoddard, 2015). Por su poder en la regulación de la conducta, los PSs tienen importantes implicancias para la psicoterapia (Bak, 2015).

Los PSs son una construcción social. Un individuo puede crear una variedad de sí mismos potenciales pero éstos derivan de las categorías salientes en su contexto sociocultural e histórico. Los modelos, imágenes y símbolos provenientes de los medios de comunicación y de los entornos sociales más próximos (e. g. la familia, la escuela) del individuo le proveen representaciones de quién podría ser en el futuro (Oyserman \& Fryberg, 2006). El ambiente físico y geográfico también tiene implicancias en la constitución de los PSs (Prince, 2014). En Argentina, los adolescentes dan mucha importancia a la construcción de su futuro desde el momento presente. La escuela y la familia son contextos con gran relevancia para dicha construcción (Molina, Gimenez, Raimundi, Leibovich \& Schmidt, 2015).

El contenido de los posibles sí mismos ha sido estudiado en otros países. En la Tabla 1 se resumen los resultados obtenidos en estos estudios. Éstos fueron llevados a cabo en países anglosajones $\mathrm{y}$, recientemente, en países asiáticos, como Hong Kong (Zhu \& Tse, 2015). Por lo tanto es necesario considerar el contenido idiográfico de los PSs de los adolescentes latinoamericanos. La posibilidad de proyección de las personas, en tanto devenir subjetivo, en contextos anglosajones puede ser diferente que aquella surgida en la idiosincrasia latinoamericana. Ya que involucra una valoración del futuro, de la posibilidad de estabilidad y previsibilidad de los roles a llevar a cabo en el futuro. Hasta ahora no se han estudiado, en Argentina, los posibles sí mismos de los adolescentes. 
Tabla 1. Estudios acerca del contenido de los PSs en adolescentes.

\begin{tabular}{|c|c|c|c|c|}
\hline Autor & Muestra & Referencia temporal & Tipo de PSs & Posibles Sí mismos \\
\hline $\begin{array}{l}\text { Oyserman \& Markus } \\
\text { (1990) }\end{array}$ & $\begin{array}{c}\text { Adolescentes norteamericanos }(n=238) \\
\text { de } 13 \text { a } 16 \text { años, población general y } \\
\text { con distintos niveles de comportamiento } \\
\text { delictivo. }\end{array}$ & Próximo año & $\begin{array}{l}\text { Esperados y temidos } \\
\text { Esperados }\end{array}$ & $\begin{array}{c}\text { Sí mismos intrapersonales } \\
\text { Sí mismos interpersonales } \\
\text { Escuela y actividades extraescolares } \\
\text { Trabajo } \\
\text { Bienes materiales } \\
\text { PSs negativos } \\
\text { Pobreza } \\
\text { Crimen } \\
\text { Drogas } \\
\text { Muerte }\end{array}$ \\
\hline Knox et al. (1998) & $\begin{array}{l}\text { Adolescentes norteamericanos }(n=212) \\
\text { de población general de } 14 \text { a } 19 \text { años. }\end{array}$ & El futuro & Deseados y temidos & $\begin{array}{c}\text { Descriptores personales según uno mismo } \\
\text { Descriptores personales según se es visto por los } \\
\text { otros } \\
\text { Apariencia física } \\
\text { Funcionamiento psicológico } \\
\text { Ocupación } \\
\text { Educación } \\
\text { Descriptores materiales y financieros } \\
\text { Intereses y hobbies } \\
\text { Deportes } \\
\text { Relaciones y funcionamiento interpersonal } \\
\text { Salud física } \\
\text { Intereses sociales/religión } \\
\text { Independencia } \\
\text { Éxito general/reconocimiento } \\
\text { Enfermedad física/muerte } \\
\text { Dependencia } \\
\text { Fracaso general/inferioridad } \\
\text { Sexualidad } \\
\text { Daño externo/victimización }\end{array}$ \\
\hline $\begin{array}{l}\text { Shepard \& Marshall } \\
\text { (1999) }\end{array}$ & $\begin{array}{l}\text { Adolescentes }(n=44) \text { de entre } 11 \text { y } 13 \\
\text { años de Canadá. }\end{array}$ & Futuro & $\begin{array}{l}\text { Deseados } \\
\text { Temidos }\end{array}$ & $\begin{array}{c}\text { Educación/aprendizaje } \\
\text { Relaciones interpersonales } \\
\text { Ideales } \\
\text { Estilo de vida } \\
\text { Trabajo significativo } \\
\text { Ocupación } \\
\text { Posesiones } \\
\text { Tiempo libre } \\
\text { Salud } \\
\text { Mortalidad } \\
\text { Seguridad/protección } \\
\end{array}$ \\
\hline $\begin{array}{l}\text { Aloise-Young et al. } \\
\qquad(2001)\end{array}$ & $\begin{array}{c}\text { Adolescentes Norteamericanos de } \\
\text { población general }(n=1606) \text { de entre } 11 \\
\text { y } 15 \text { años. }\end{array}$ & Próximo año & Deseados y temidos & $\begin{array}{c}\text { Intrapersonal }^{\mathrm{a}} \\
\text { Interpersonal } \\
\text { Trabajo/empleo } \\
\text { Crimen } \\
\text { Escolar o actividades extracurriculares } \\
\text { Bienes materiales } \\
\text { Salud } \\
\text { Abuso de substancias } \\
\text { General } \\
\end{array}$ \\
\hline \multirow[t]{4}{*}{ Oyserman (2004) } & \multirow{4}{*}{$\begin{array}{l}\text { Basado en las investigaciones de } \\
\text { Oyserman con diferentes muestras de } \\
\text { adolescentes norteamericanos }\end{array}$} & \multirow{4}{*}{ Próximo año y adultez } & Esperados y temidos & $\begin{array}{c}\text { Logros escolares y en otras actividades } \\
\text { Relaciones interpersonales } \\
\text { Rasgos de personalidad } \\
\text { Físico y relativo a la salud } \\
\text { Material/estilo de vida }\end{array}$ \\
\hline & & & Esperados & PSs negativos \\
\hline & & & $\begin{array}{l}\text { Temidos (próximo } \\
\text { año) }\end{array}$ & Comportamiento desviado o de riesgo \\
\hline & & & Temidos (adulto) & Delincuencia \\
\hline McClelland (2011) & $\begin{array}{l}\text { Adolescentes }(n=52) \text { de } 13 \text { a } 16 \text { años } \\
\text { de Inglaterra. }\end{array}$ & Futuro & Deseados y temidos & $\begin{array}{c}\text { Éxito/fracaso general } \\
\text { Ocupación } \\
\text { Interpersonal/relaciones } \\
\text { Material/financiero Educacional/académico } \\
\text { Salud general } \\
\text { Salud psicológica } \\
\text { Apariencia general } \\
\end{array}$ \\
\hline Zhu \& Tse (2015) & $\begin{array}{l}\text { Adolescentes }(n=3078) \text { de } 12 \text { a } 20 \text { años } \\
\text { de Hong Kong. }\end{array}$ & Futuro & Deseados y temidos & $\begin{array}{c}\text { Escuela } \\
\text { Profesión } \\
\text { Logros } \\
\text { Interpersonal } \\
\text { Rasgos de personalidad } \\
\text { Salud/física } \\
\text { Bienes materiales y estilo de vida } \\
\text { Alcohol y drogas } \\
\text { Delincuencia }\end{array}$ \\
\hline
\end{tabular}

Nota. PSs $=$ posibles sí mismos.

${ }^{a}$ Siete de las categorías son tomadas del estudio de Oyserman y Markus (1990) y dos son desarrolladas para este estudio. 
Por otra parte, los PSs pueden ser distantes en el tiempo como el sí mismo adulto de un adolescente o más próximos como en quién se va a convertir el próximo año (Oyserman \& Fryberg, 2006). En estudios anteriores se consideró como punto de referencia la adultez, el futuro o el año próximo (ver Tabla 1). En este estudio se decidió indagar los PSs de los adolescentes al terminar el secundario. Dicho suceso constituye una transición vital significativa en la que el adolescente entra a un nuevo ambiente y que se encuentra asociada al inicio de la vida adulta (Malin, Reilly, Quinn $\&$ Moran, 2014). Se la eligió por ser un punto de referencia definido a diferencia de puntos de referencia como "ser adulto" o el "futuro", que son inespecíficos y que pueden ser interpretados de manera diferente por cada adolescente.

En síntesis, los posibles sí mismos cumplen un rol capital en la exploración de la identidad de los adolescentes, asimismo tienen un importancia central para la elección de acciones entre las conductas posibles y para poder llevar adelante dichas acciones. La influencia del contexto sociocultural en los PSs plantea la relevancia de estudiar el contenido de los PSs antes de aplicarlos al estudio o intervención en una nueva población (Zhu \& Tse, 2015). El estudio de los PSs en adolescentes argentinos, permite conocer las áreas que más los motivan y mayor poder tienen en la regulación de su comportamiento, y los dominios que componen la exploración de su identidad. Asimismo, permite conocer aquellas áreas en las que no se proyectan a futuro $\mathrm{y}$ en las que puede ser relevante intervenir.

La importancia de estudios de este tipo radica en otorgar validez ecológica a conceptos estudiados en otros contextos, de relevancia teórica indudable, pero sobre los cuales desconocemos su contenido particular en nuestro contexto específico. De esta forma, el objetivo central del trabajo es describir las características de los posibles sí mismos en adolescentes de una escuela privada de la Ciudad de Buenos Aires.

Rev.latinoam.cienc.soc.niñez juv 15 (1): 455-470, 2017

http://revistalatinoamericanaumanizales.cinde.org.co

DOI: $10.11600 / 1692715 \times .1512901102015$

\section{Método}

El presente estudio es de tipo exploratoriodescriptivo con un diseño transversal (Hernández Sampieri, Fernández-Collado \& Baptista Lucio, 2010).

\section{Participantes}

La muestra estuvo compuesta por adolescentes $(n=72)$ de ambos sexos (mujeres $=69.4 \%$ ) que asistían a una escuela, privada y católica, de enseñanza media de la Ciudad de Buenos Aires (edad $M=15.06 ; S D=1.56$ ). Un $70.4 \%$ de los adolescentes pertenecían a hogares biparentales. Un $48.6 \%$ de las madres y el $40.3 \%$ de los padres tenía estudios universitarios completos. La mayoría de los padres tiene un empleo estable (madres = $90.0 \%$; padres $=88.7 \%$ ).

\section{Instrumentos}

-Cuestionario

socio-demográfico: cuestionario especialmente diseñado para caracterizar a la muestra en función de variables sociodemográficas (e. g. edad, nivel educativo de los padres, composición familiar).

-Cuestionario de los posibles sí mismos. Autores: Oyserman (2004). Adaptación: Molina (2014). Es un cuestionario de preguntas abiertas en el que se pide al adolescente que realice un listado de cuatro posibles sí mismos esperados, deseados y temidos.

\section{Procedimiento}

Se contactó a las autoridades de la escuela en la que se realizó el estudio. Se les explicó el propósito del proyecto. La participación de los adolescentes fue voluntaria, anónima y confidencial. Se solicitó el consentimiento escrito de los padres indicando que aceptaban que sus hijos participaran en el estudio. Los adolescentes respondieron los cuestionarios en forma grupal. La consigna fue explicada por los investigadores.

Este proyecto cuenta con el aval del Comité de Ética de la Facultad de Psicología de la Universidad de Buenos Aires. 


\section{Análisis de datos}

Se realizó un análisis de contenido temático a las respuestas de los adolescentes, para lo que se siguieron los lineamientos propuestos por Strauss y Corbin (1990).

Tres codificadores analizaron las respuestas de los adolescentes sobre sus PSs esperados. A partir del consenso entre los codificadores se estableció un libro de códigos. Dicho libro de códigos fue aplicado de forma independiente por dos codificadores para analizar las respuestas para los PSs deseados y temidos con un 76\% de concordancia. Se discutieron las categorías en las que no hubo concordancia hasta llegar a un consenso. A partir de las categorías identificadas se generaron categorías globales o temas que integran a las categorías de primer nivel.

En último lugar, como criterios de validez en metodología cualitativa, un investigador, externo al proceso ya realizado, fue convocado para revisar y auditar las categorizaciones realizadas. Este procedimiento se orientó a establecer la "credibilidad", "dependencia" y "confirmabilidad" de los datos (HernándezSampieri et al., 2010).

Se realizó un conteo de la frecuencia con que las categorías de primer nivel y globales fueron informadas por los adolescentes. Para esto se estimó el porcentaje de sujetos que mencionó la categoría, por lo menos, una vez. Para conocer la diversidad del contenido de los PSs expresados por los adolescentes, se calculó la cantidad de temas a los que hacían referencia los posibles sí mismos de cada adolescente.

\section{Resultados}

\section{Descripción de los dominios y áreas de los PSs de los adolescentes}

En la Tabla 2 se presenta un resumen de los dominios y áreas encontrados para los PSs de los adolescentes. A continuación se describe su contenido en cada tipo de PSs: esperados, temidos y deseados. 
Tabla 2. Contenido y prevalencia de las áreas y dominios de los PSs encontrados.

\begin{tabular}{|c|c|c|c|c|}
\hline Dominio & Descripción & $\begin{array}{c}\text { PSs Esperados } \\
n(\%)\end{array}$ & $\begin{array}{c}\text { PSs Temidos } \\
n(\%)\end{array}$ & $\begin{array}{c}\text { PSs Deseados } \\
n(\%)\end{array}$ \\
\hline I. Personal & & $37(51.4)$ & $47(65.3)$ & $33(45.8)$ \\
\hline 1. Ético/moral & $\begin{array}{l}\text { Valores éticos y morales que los adolescentes } \\
\text { esperan, temen o desean tener. }\end{array}$ & $9(12.5)$ & $21(29.2)$ & $10(13.9)$ \\
\hline 2. Responsabilidad & $\begin{array}{l}\text { Descriptores personales que hacen referencia } \\
\text { a la madurez, dedicación y compromiso con } \\
\text { las propias actividades }\end{array}$ & $25(34.7)$ & $20(27.8)$ & $9(12.5)$ \\
\hline 3. Energía/vitalidad & $\begin{array}{l}\text { Descriptores personales que dan cuenta de la } \\
\text { fuerza y empuje de la persona }\end{array}$ & $6(8.3)$ & $4(5.6)$ & $3(4.2)$ \\
\hline 4. Sociabilidad & $\begin{array}{l}\text { Descriptores personales que hacen referencia } \\
\text { a la forma en que la persona se relaciona con } \\
\text { los demás }\end{array}$ & $11(15.3)$ & $18(25.0)$ & $12(16.7)$ \\
\hline 5. Competencias & $\begin{array}{l}\text { Competencias generales y específicas que } \\
\text { caracterizan a los PSs }\end{array}$ & $7(9.7)$ & $3(4.2)$ & $9(12.5)$ \\
\hline 6. Confianza en sí mismos & $\begin{array}{l}\text { Descriptores personales referidos a la } \\
\text { seguridad o confianza personal }\end{array}$ & $6(8.3)$ & $4(5.6)$ & $4(5.6)$ \\
\hline 7. Autonomía & $\begin{array}{l}\text { Independencia o autonomía económica, } \\
\text { emocional o afectiva }\end{array}$ & $11(15.3)$ & $10(13.9)$ & $6(8.3)$ \\
\hline 8. Conducta problemática & Conductas de riesgo o disruptivas & $0(0.0)$ & $5(6.9)$ & $0(0.0)$ \\
\hline II. Físico & & $5(6.9)$ & $2(2.8)$ & $2(2.8)$ \\
\hline 1. Apariencia física & $\begin{array}{l}\text { Características del cuerpo, cambios en el } \\
\text { cuerpo. }\end{array}$ & $2(2.8)$ & $0(0.0)$ & $2(2.8)$ \\
\hline 2. Deportivo & $\begin{array}{l}\text { Aptitud para los deportes y relación con } \\
\text { la actividad deportiva (si se continúa o no } \\
\text { practicándola). }\end{array}$ & $4(5.6)$ & $2(2.8)$ & $0(0.0)$ \\
\hline III. Académico /ocupacional & & $52(72.2)$ & $50(69.4)$ & $53(73.6)$ \\
\hline $\begin{array}{l}\text { 1. Estudio e intereses } \\
\text { académicos }\end{array}$ & $\begin{array}{l}\text { Relación de los adolescentes con el estudio } \\
\text { y las cosas que quieren aprender. Incluye los } \\
\text { intereses y aspiraciones, y el compromiso que } \\
\text { espera, desea o teme tener con el estudio. }\end{array}$ & $25(34.7)$ & $22(30.6)$ & $18(25.0)$ \\
\hline 2. Profesión/ocupación & Profesiones y ocupaciones específicas & $30(41.7)$ & $17(23.6)$ & $33(45.8)$ \\
\hline 3. Trabajo & $\begin{array}{l}\text { Relación de los adolescentes con el trabajo. } \\
\text { Si piensan que van a trabajar o no. Cuestiones } \\
\text { generales del trabajo. }\end{array}$ & $5(6.9)$ & $20(27.8)$ & $10(13.9)$ \\
\hline 4. Estilo de vida & $\begin{array}{l}\text { Características de la forma de vida que } \\
\text { esperan, temen o desean tener. }\end{array}$ & $9(12.5)$ & $7(9.7)$ & $13(18.1)$ \\
\hline IV. Social & & $18(25.0)$ & $7(9.7)$ & $18(25.0)$ \\
\hline 1. Familia y seres queridos & $\begin{array}{l}\text { Referencias a las relaciones familiares y a } \\
\text { relaciones afectivas inespecíficas. }\end{array}$ & $13(18.1)$ & $4(5.6)$ & $15(20.8)$ \\
\hline 2. Pares & Relación con los amigos o compañeros. & $7(9.7)$ & $3(4.2)$ & $6(8.3)$ \\
\hline V. Satisfacción y logros & & $17(23.6)$ & $13(18.1)$ & $23(31.9)$ \\
\hline 1. Felicidad & $\begin{array}{l}\text { Referencias a ser felices y estar satisfechos } \\
\text { con sus vidas. }\end{array}$ & $5(6.9)$ & $1(1.4)$ & $7(9.7)$ \\
\hline 2. Éxito & $\begin{array}{l}\text { Éxito y satisfacción con los logros. Incluye el } \\
\text { éxito social, ser reconocido o destacado entre } \\
\text { los demás. }\end{array}$ & $7(9.7)$ & $5(6.9)$ & $10(13.9)$ \\
\hline 3. Metas & $\begin{array}{l}\text { Referencias a la claridad de metas y objetivos, } \\
\text { la motivación para seguir esas metas y deseos } \\
\text { de auto-superación. Cuestiones vinculadas } \\
\text { al proceso de llevar adelante un objetivo } \\
\text { (saber tomar decisiones y aprovechar las } \\
\text { oportunidades). }\end{array}$ & $8(11.1)$ & $7(9.7)$ & $11(15.3)$ \\
\hline 4. Ilusiones y sueños & Deseos o aspiraciones lejanos e idealizados & $0(0.0)$ & $0(0.0)$ & $2(2.8)$ \\
\hline VI. Complejidad & & $10(13.88)$ & $3(4.17)$ & $6(8.33)$ \\
\hline
\end{tabular}

Nota. PSs $=$ posibles sí mismos. 
I. Personal: PSs que describen características personales o rasgos de personalidad.

A. Ético/moral: Los adolescentes esperan (PSs esperados) ser personas que poseen $y$ se comportan de acuerdo a valores morales y éticos ("Una persona con muchos valores adquiridos", "Buena persona”, "Honesto"). Temen ser (PSs temidos) malas personas, mentirosos, deshonestos e irrespetuosos. También hacen mención a la falta de gratitud y el perdón ("Mala persona en todo tipo de casos", "Hipócrita”). Los PSs deseados coinciden en gran medida con la que creen que van a ser ("Una persona incorruptible", "Tener buenos modales").

B. Competencias: Los adolescentes esperan ser personas capaces y tener habilidades cognitivas como: ser creativo, ser prolijo y ordenado ("Una persona capaz", "Una persona más inteligente”). Los adolescentes temen a la falta total de competencias. No hacen referencias a competencias específicas ("Mediocre", "Inútil"). Los PSs deseados coinciden con los esperados. Desean tener altas capacidades y habilidades cognitivas específicas ("Talentosa”, "Creativa”).

C. Responsabilidad: Los adolescentes esperan ser trabajadores, comprometidos, perseverantes ("Más maduro", "Dedicada, perseverante"). Temen tener falta de dedicación y compromiso en sus actividades ("Una persona vaga", "Alguien que no le importe nada"). Los PSs deseados coinciden con los esperados

D. Energía/vitalidad: Los adolescentes esperan y desean ser personas con fuerza $\mathrm{y}$ empuje; ser activos, alegres, entusiastas y divertidos ("Entusiasta", "Impaciente"). Temen a la falta de energía y vitalidad ("Aburrida", "Malhumorada", "Negativa").

E. Sociabilidad: Los PSs esperados hacen referencia a la disposición a interactuar con los demás, a la falta de temor o inhibición en la relación con los demás, y a la disposición a ayudar a otros ("Amigable", "Extrovertida", "Solidario"). Los PSs temidos son la contracara de los esperados: temen tener poca disposición para relacionarse, tener miedo y desconfianza hacia los otros y a la falta de disposición a ayudar a los demás e, incluso, a agredirlos ("Una persona solitaria", "Tímida", "Agresivo"). También temen tener características que generan el rechazo de los demás ("Insoportable"). En general, los PSs deseados coinciden con los PSs esperados, pero se suman a éstos el ser agradable a los demás ("Carismática").

F. Autonomía: Los adolescentes piensan que van a ser independientes, tener su propio hogar, tomar sus propias decisiones y no necesitar de los demás ("Voy a tomar mis propias decisiones", "Voy a ser independiente, teniendo mi propio hogar"). No quieren depender de otros, hacer lo que los demás quieren, ser una carga ("Ser una carga para alguien", "Hacer lo que los demás quieren"). Los PSs deseados coinciden en su contenido con los PSs esperados.

G. Confianza en sí mismo: Los adolescentes creen que van a ser seguros y confiar en ellos mismos ("Una chica más confiada", "Segura de mi misma"). El exceso de autoconfianza es temido por los adolescentes ("Creérmela"). En el extremo opuesto, los adolescentes temen a la falta de decisión ("Indecisa"). Los adolescentes desean tener características que denotan autoconfianza: ser decididos, audaces y creer en ellos mismos ("Decidida", “Audaz").

H. Conducta problemática: Específicamente en los PSs temidos aparece el temor a desarrollar conductas relacionadas con el consumo excesivo de sustancias psicoactivas, con la participación en peleas y el descontrol ("Un chico que pelee", "Voy a emborracharme todos los días", "Voy a descontrolarme siempre”).

II. Académico/ocupacional: PSs que describen las ocupaciones y cuestiones vinculadas a ellas.

A. Profesión/ocupación: Como parte de sus PSs esperados, los adolescentes hacen referencia a distintos tipos de ocupaciones y profesiones. En su mayoría son profesiones que requieren de estudios terciarios o universitarios, también hacen mención a carreras artísticas o deportivas. Algunos adolescentes hacen referencia a sus intereses ocupaciones más que a una profesión definida ("Médico", "Cantante 
o actriz", "Tenista", "Psicóloga porque me gusta ayudar mucho a los demás"). Algunas de las profesiones u ocupaciones temidas por los adolescentes coinciden con las esperadas, sin embargo, otras aparecen exclusivamente entre los PSs temidos ("Ama de casa", "Taxista" "Cartonera", "Masajista"). En los PSs deseados aparecen en mayor medida que en los esperados las profesiones deportivas.

B. Trabajo: Los adolescentes esperan tener trabajo o buscar trabajo ("Voy a estar buscando trabajo”). Como parte de los PSs temidos aparece, en primer lugar, la falta de trabajo. También hacen referencias a características del trabajo que no quieren tener. Por último, hacen referencia a tener un mal desempeño laboral ("Una persona desempleada", "Una persona que trabaje en una oficina todo el día", "Ser malo en mi trabajo"). En los PSs deseados, además del deseo de tener trabajo, aparece el deseo de que éste sea bueno y estable ("Tener un trabajo bueno y estable").

C. Estudios e intereses académicos: En los PSs esperados, los adolescentes hacen referencia al compromiso con el estudio, continuar estudiando después de terminar el secundario, la elección vocacional y al estudio como un medio para el desarrollo profesional y vocacional ("Más estudioso", "Elegir la carrera que más me guste", "Estudiaría actuación o canto para llegar a ser una actriz y gran cantante"). Asimismo, hacen referencia a los deseos de aprender y de poseer conocimientos ("Una persona culta con muchos intereses", "Voy a conocer cosas nuevas y me va a interesar aprender").

Los adolescentes temen no seguir estudiando después del secundario, no estar comprometidos con el estudio, no tener definida la vocación y no tener conocimientos en general ("Alguien que apruebe con la nota mínima", "Sin conocimientos culturales y educativos"). Por otro lado, aparece el temor a no poder dedicar tiempo a otras actividades e intereses más allá del estudio ("Concentrada sólo en el estudio").

El contenido de los PSs deseados coincide con el de los PSs esperados con la diferencia de que los adolescentes no hacen referencia a la adquisición de conocimientos por sí misma sino que se vincula siempre con el desarrollo de la vocación y profesión.

D. Estilo de vida: Los adolescentes esperan tener un estilo de vida en el que puedan viajar, disfrutar del tiempo libre, realizar actividades recreativas y tener ciertos bienes materiales ("Tener mi auto", "Alguien que dedique tiempo a sus gustos”). Temen ser alguien que no tenga vivienda, a quien le falte el sustento material y el dinero necesario para vivir y progresar ("Ganar muy poco dinero para progresar", "Vivir en la calle [intentaría salir]”). El contenido de los PSs deseados es similar al de los esperados. Sin embargo, los adolescentes ponen más énfasis en la posesión de riquezas materiales y de la fama. Al igual que en los PSs esperados, desean viajar, vivir en otro país, realizar actividades recreativas y disponer de lo necesario para el autosustento ("Tener una mansión y vivir bien", "Ser famosa”).

III. Físico.PSs que describen características de la apariencia y aptitudes físicas de los adolescentes.

A. Apariencia física: Son muy pocos los adolescentes que hacen referencia a este dominio. Los que lo hacen se refieren a su estatura y a la posibilidad de que su cuerpo no cambie, lo cual es vivido con disgusto ("Igual de cuerpo, el cual no me gusta", "Más alto"). No hacen referencia en esta categoría a la forma en que temen ser. Desean ser más altos, más delgados y bellos ("Más alta", "Más flaca y bonita").

B. Deportivo: Los adolescentes esperan poder seguir practicando deportes. Algunos creen que van a ser malos en los deportes ("Continuar con la actividad deportiva", "Mala deportista"). Temen abandonar la actividad deportiva ("No terminar los deportes que me gustan"). Desean dedicarse a los deportes ("Deportista").

IV. Social: PSs que describen las relaciones interpersonales.

A. Relación con los pares: Los adolescentes esperan tener muchos amigos, estar unidos a ellos, recibir su apoyo y mantener el contacto con los amigos que ya tienen ("Con amigos al pié del cañón con los 
con que mantengo contacto y salgo", "Alguien con muchos amigos"). Temen no tener amigos y perder el contacto con los que tienen ("Perder el contacto con mis amigos o las personas que quiero", "No tener amigos"). Desean mantener los amigos del secundario ("Seguir con los amigos de la secundaria", "Seguir hablando con mis ex compañeros").

B. Familia y seres queridos: Los adolescentes esperan ser parte de una familia, poder formar una familia propia, y mantenerse unidos a su familia de origen y seres queridos en general ("Parte de una familia", "Voy a formar una familia con cuatro hijos", "Muy unida a las personas que quiero"). Temen no tener una familia o poder formar pareja ("No tener familia", "Soltero") El contenido de los PSs deseados es el mismo que en PSs esperados.

V. Satisfacción y logros: PSs que describen la satisfacción general, las aspiraciones y los logros de los adolescentes.

A. Felicidad: Los adolescentes esperan y desean ser felices y estar satisfechos con sus vidas ("Satisfecha por como llevo mi vida", "Ser feliz"). Temen ser infelices ("No quiero ser infeliz").

B. Metas personales: Los adolescentes piensan que cuando terminen el secundario van a ser personas con metas y objetivos claros, con motivación para seguir esas metas y deseos de autosuperación ("Voy a ser una persona con objetivos claros", "Cuando me proponga algo lo voy a cumplir"). Temen no tener metas, objetivos o proyectos. También hacen referencia a no saber aprovechar las oportunidades, tomar malas decisiones, o manejarse mal en la vida ("Una persona que no le guste tener un futuro", "No quiero ser una persona sin objetivos y metas para mi futuro"). Desean tener proyectos definidos, luchar por esas metas y cumplirlas ("Seguir teniendo propósitos para mi vida", "Una luchadora por lo que quiero").

C. Éxito general: Esperan ser exitosos, triunfar, llegar lejos en sus actividades, estar satisfechos por sus logros ("Poder triunfar", "Una personas feliz por sus logros"). Temen fracasar, no tener futuro y a la falta de reconocimiento social ("Una mujer sin futuro", "No ser nadie en el mundo social"). Desean tener éxito y progresar en la vida ("Exitosa en lo que me proponga", "Progresar en la vida").

D. Sueños e ilusiones: Específicamente en los PSs deseados aparecen deseos o aspiraciones platónicas, es decir, lejanos e idealizados. Involucran la fama y reconocimiento social ( "Ir al "the x factor" para ganar y ser una gran cantante", "Estar en "Mastercheff"”).

VI. Complejidad: Algunos adolescentes articulan más de uno de los dominios anteriores en una síntesis totalizadora que integra varios aspectos del posible sí mismo o realizan una mayor elaboración de sus PSs en un mismo dominio. En algunos casos esto implica una interfaz entre dos microcontextos. Puede ser tanto que el desempeño en un contexto favorezca el de otro ("Una persona con una buena formación secundaria la cual trabaje") como la idea de poder compatibilizar los roles en dos microsistemas diferentes ("Me veo haciendo danza a la par del estudio", "Voy a trabajar para ganar plata mientras estudio", "Un chico que se concentre en sus estudios pero que le de mucha importancia a sus amigos"). En otros casos, se articulan el éxito o el progreso con el desempeño en otros dominios ("Un fracaso, tardar mil años y fracasar en la carrera que elija").

\section{Prevalencia del contenido de los PSs}

En la Tabla 2 se presenta la frecuencia con que los adolescentes generaron PSs en cada uno de los dominios y áreas descriptos. El área más prevalente es la académico/ocupacional. Dentro de esta área, para los PSs esperados y deseados, el dominio más prevalente es el de la profesión/ocupación. El dominio de los estudios e intereses académicos y el trabajo son los más prevalentes en los PSs temidos.

El segundo área más prevalente es la personal. Entre los PSs esperados, el dominio más prevalente es el de la responsabilidad. Entre los PSs temidos, es el ético/moral. El dominio de la sociabilidad es el más prevalente entre los PSs deseados.

\section{Diversidad temática en los PSs de los adolescentes}

En la Tabla 3 se presenta el porcentaje de adolescentes que generó posibles sí mismos en entre una a cinco de las áreas encontradas. 
La mayoría de los adolescentes generó PSs en una o dos áreas de PSs. En los PSs esperados y deseados un porcentaje considerable de adolescentes generó PSs en tres áreas. Son escasos los adolescentes que generaron PSs en cuatro de las áreas y sólo uno de ellos generó PSs deseados en todas las áreas.

Tabla 3. Número de áreas en las que los adolescentes generaron PSs.

\begin{tabular}{cccc}
\hline $\mathrm{N}^{\circ}$ de áreas & $\begin{array}{c}\text { PSs Esperados } \\
n(\%)\end{array}$ & $\begin{array}{c}\text { PSs Temidos } \\
n(\%)\end{array}$ & $\begin{array}{c}\text { PSs Deseados } \\
n(\%)\end{array}$ \\
\hline 1 & $34(47.9)$ & $33(45.8)$ & $24(36.4)$ \\
2 & $18(25.7)$ & $33(45.8)$ & $25(37.9)$ \\
3 & $17(24.3)$ & $4(5.6)$ & $14(21.2)$ \\
4 & $2(2.9)$ & $2(2.8)$ & $2(3.0)$ \\
5 & $0(0.0)$ & $0(0.0)$ & $1(1.5)$ \\
\hline
\end{tabular}

Nota. PSs $=$ posibles sí mismos.

\section{Discusión}

El objetivo del presente estudio fue explorar cómo se perciben a sí mismos en el futuro un grupo de adolescentes de la Ciudad de Buenos Aires (Argentina). El análisis cualitativo de los datos reveló que los adolescentes proyectan su visión de sí mismos en el futuro en cinco grandes áreas. En el área personal hacen referencia a los valores éticos y morales según los que esperan actuar, a su dedicación y compromiso con las propias actividades, a su fuerza y empuje; a sus conductas en la relación con los demás, a sus competencias personales; a la seguridad en sí mismos, a su independencia económica y emocional, y a las conductas disruptivas que no quieren tener. El área académico/ocupacional refleja las expectativas de los adolescentes con respecto a ocupación, los logros materiales y su desempeño en el ámbito del estudio y la adquisición de conocimientos. El área física refleja la imagen corporal que proyectan a futuro y su relación con los deportes. El área social se conformó por los dominios de la relación con la familia y seres queridos, y los pares. Por último, el área de la satisfacción y logros refleja la posibilidad de ser felices, la claridad de sus objetivos y la motivación para seguir sus metas, el éxito en sus actividades y el reconocimiento social, y los deseos o aspiraciones que parecen lejanos y difíciles de alcanzar.

En general, el contenido encontrado coincide con el de los estudios presentados en la Tabla 1. Sin embargo, se encontraron algunas diferencias. Por un lado, los adolescentes no hacen referencia a la salud física, psicológica o general, la seguridad o protección, o la mortalidad que sí fueron expresadas por adolescentes de otras muestras (Aloise-Young et al., 2001, Knox et al., 1998, McClelland, 2011, Oyserman \& Markus, 1990, Oyserman, 2004, Shepard \& Marshall, 1999, Zhu \& Tse, 2015). Pareciera que los dominios expresados por los adolescentes de este estudio están claramente anclados en el momento actual, expresando inquietudes propias de esta etapa evolutiva (Oyserman \& James, 2009). Estos resultados son coherentes con la idea de la adolescencia como estapa signada por la vitalidad, la actividad y las posibilidades (Barber, Abbott, Blomfield \& Eccles, 2009).

Los adolescentes del presente estudio, tampoco hacen referencias al crimen $y$ delincuencia que sí aparecen en otros trabajos (Aloise-Young et al., 2001, Oyserman, 2004, Oyserman \& Markus, 1990, Zhu \& Tse, 2015). Es importante señalar que muchos de los estudios citados trabajaron con adolescentes institucionalizados por comportamiento delictivo.

Uno de los dominios emergentes en nuestro estudio es el de las metas. Resulta interesante que los adolescentes no se plantean sólo la meta, el llegar a ser de determinada manera, sino que también valoran ser personas que continúan planteándose metas y que se comprometen para lograrlas. En este sentido, el posible sí mismo con metas plantea un dinamismo, en el que el 
conseguir las metas deseadas no es un momento cristalizado, sino que se resignifica y se vuelve al mismo movimiento que empuja a la persona a abrazar nuevos desafíos, en un continuo. En el estudio del bienestar se ha encontrado que es el proceso de esforzarse tras metas, más que el logro de metas por sí mismo, lo que es crucial para la felicidad y para la afectividad positiva (Watson, 2002). En un trabajo con adolescentes de la Ciudad de Buenos Aires se muestra la importancia de las metas para los adolescentes, las cuales tienen un papel central en la asunción de desafíos (Raimundi, Molina, Gimenez \& Minichiello, 2014).

Asimismo, un tema emergente en nuestro trabajo es que algunos adolescentes presentan una articulación e integración entre sus PSs que complejiza su percepción de sí mismos en el futuro. Esta categoría puede ser pensada desde el Paradigma de la Complejidad en tanto proyecta una visión unificadora a partir de la integración de todos los elementos y dimensiones que lo constituyen, siendo una visión multidimensional e integradora que establece puentes entre los distintos niveles de organización del sistema generando enfoques integrados del conocimiento (Núñez \& Romero, 2003).

Al igual que en otros contextos, la mayoría de los adolescentes generaron PSs en el área académico ocupacional (Marshall, Young, Domene, \& Zaidman-Zait, 2008, Oyserman \& James, 2009, Zhu \& Tse, 2015). Dentro de esta área, los PSs más prevalentes se refieren a la profesión/ocupación y a los estudios e intereses académicos. Esto es coherente con lo que se encontró al preguntar a los adolescentes por su futuro en general, el contenido principal de la imagen que tienen de su futuro hace referencia al estudio y la ocupación (Molina et al., 2015).

Entre los PSs temidos también aparece el dominio del trabajo con una alta prevalencia. La inestabilidad laboral que caracterizó a la historia socio-económica reciente en nuestro país, y en la mayoría de los países latinoamericanos, presenta al campo del trabajo como crítico para proyectarse. Esta historia muy posiblemente no fue vivida directamente por estos adolescentes, por su edad, pero el contexto en el que nacieron trajo consigo esta resignificación del campo del trabajo como inestable y poco previsible sobre el cual proyectarse.

Al comparar los tipos de PSs se observa que los tres tipos (esperados, temidos y deseados) se expresaron en las mismas áreas y dominios. Salvo en el caso del dominio de conducta problemática, que sólo emergió como PSs temido, e ilusiones y sueños, que sólo emergió como un PSs deseados.

Sin embargo, en algunos dominios se observan diferencias en el contenido según el tipo de PSs. En el dominio de la profesión/ ocupación, en los PSs esperados aparece un listado de profesiones clásicas y en los PSs deseados muchos adolescentes pudieron desarrollar más sus intereses o, quizás, sus deseos más profundos, más allá de lo "socialmente esperado". Es posible que esto exprese la influencia del contexto sociocultural en el que viven los adolescentes. Como se mencionó anteriormente, la inestabilidad económica ha sido predominante en nuestro contexto y las profesiones "clásicas" pareciera que son las más "recomendadas" para subsistir, mientras otras, sobre todo, las artísticas y las deportivas no son las más "alentadas" como profesiones por la escuela y por los padres. Por otra parte, en esta muestra, en particular, esto puede tener que ver con la historia familiar, ya que muchos de los participantes son hijos de profesionales.

Por otra parte, entre los PSs deseados y temidos hay una gran diferencia en este dominio. Los PSs deseados involucran la realización vocacional asociada, además, a logros materiales y reconocimiento social ("un creador de joyas cinematográficas", "ser la más grande veterinaria", "jugar en la NBA"). Las PSs temidos incluyen oficios de servicio, con un menor reconocimiento social y menores niveles de ingresos salariales ("taxista" o "mucama"); e incluso situaciones que connotan marginación y exclusión social ("prostituta", "Cartonera").

Asimismo, en el dominio estilo de vida en los PSs deseados la adquisición de riquezas y fama se destaca, mientras que en los temidos surge el temor a situaciones extremas de falta de recursos y sustento (e. g. "estar tirado en la calle", "vivir bajo un puente"). 
En cuando a la diversidad de áreas en los PSs, la mayoría de los adolescentes generó PSs en una o dos áreas. La diversidad de áreas en los PSs tiene consecuencias motivacionales. Los PSs son más efectivos en su función de autorregulación cuando proveen imágenes concretas y detalladas (Oyserman \& James, 2009). Los PSs están fuertemente influenciados por los factores socioculturales. En Argentina, la educación secundaria está orientada en gran medida a la preparación para insertarse en el mundo profesional a través de la continuación de los estudios (universitarios o terciarios) o de la inserción en el mercado laboral. Por esta razón no es extraño que la mayoría de los adolescentes hayan podido generar PSs en un sólo área y que el área más prevalente sea la académico/ocupacional. Sin embargo, esto señala la importancia de alentarlos a plantearse y explorar una mayor diversidad de posibles sí mismos.

Durante la adolescencia y la adultez emergente los jóvenes deben ser animados a participar en una búsqueda amplia y variada de propósito, y también deben ser alentados a centrarse en cómo planean avanzar hacia sus propósitos en la vida (Bronk, Hill, Lapsley, Talib \& Finch, 2009). Esta idea implica pensar a la adolescencia como etapa llena de potencial, y no de patología y desviación; implica alentar a buscar alternativas diversas y validarlas, no sólo impulsar destinos normalizantes relacionados con el deber ser. Explorar que aquello que se disfruta también puede convertirse en trabajo. Los adolescentes necesitan oportunidades para lograr desafíos así como resolver cuestiones vinculadas con su identidad, desarrollar una creciente autonomía y adquirir experiencias necesarias para los roles adultos de trabajo (Barber et al., 2009). Los adolescentes de Buenos Aires dan gran importancia a la construcción de su futuro desde el momento presente. Refieren que tanto sus proyectos a futuro como su actividad actual (el estudio) son claves para su desarrollo como adultos. Asimismo, la familia y la escuela aparecen como contextos con un gran potencial para desarrollar ese futuro (Molina et al., 2015).

El objetivo de este trabajo fue estudiar los PSs desde la perspectiva de un grupo de particular interés: los adolescentes. Para ello se trabajó con un grupo que concurre a una escuela secundaria privada de la Ciudad de Buenos Aires. Asimismo, se trató de una escuela religiosa, católica. Sería interesante comparar los PSs de estos adolescentes con los de adolescentes de escuelas públicas, de escuelas laicas o de otras religiones o, incluso, adolescentes que no estén escolarizados. Estudios llevados a cabo en otros países muestran que existen diferencias en los PSs de adolescentes de nivel socioeconómico bajo o que pertenecen a grupos minoritarios (Oyserman, Bybee \& Terry, 2006). Adolescentes en contextos de vulnerabilidad psico-social aportarían otros contenidos relevantes para poder pensar sus posibilidades de proyección a futuro. Por otro lado, la socialización por parte de una escuela religiosa, puede tener características específicas marcadas por su orientación hacia la ética de cada religión.

Una cuestión relevante para tener en cuenta es la edad de los participantes y la distancia temporal. El punto temporal de referencia utilizado no tiene el mismo significado para los adolescentes que están iniciando el secundario que para los que lo están terminando. El punto de referencia de los PSs no importa por su distancia cronológica sino de cuán lejano o próximo es percibido desde el punto de vista psicológico (Oyserman \& James, 2009). Futuras investigaciones deberán replicar este estudio considerando las diferencias en función de la edad.

Futuras investigaciones deberán profundizar en el estudio de los PSs recurriendo a diferentes técnicas y por medio de la triangulación de métodos. La combinación de enfoques cuali-cuantitativos permitiría dar cuenta de este constructo en toda su complejidad (Velandia-Morales, 2008). Por otro lado, se alienta a explorar cuáles son los antecedentes y consecuencias de la forma en que los adolescentes latinoamericanos se perciben a sí mismo en el futuro, así como a desarrollar estrategias para intervenir sobre ellos.

Otra de las limitaciones de este estudio es que no se estudiaron los PSs en varones y mujeres por separado. Hay estudios que señalan que los PSs difieren en función del sexo 
(Oyserman \& Fryberg, 2006 para una revisión). En futuras investigaciones será necesario ver si estas diferencias se encuentran en los adolescentes argentinos.

Más allá de estas limitaciones, este estudio permitió describir los posibles sí mismos de un grupo de adolescentes con características socioculturales particulares. Esta primera aproximación al tema, nos permite conocer cuáles son las áreas en las cuales los adolescentes proyectan su autoconcepto a futuro. Cómo piensan que van a ser, cómo temen ser y cómo desean ser en un momento particular de su futuro, que implica una transición vital significativa: el terminar la escolaridad. Conocer la diversidad de dominios y la complejidad de esta proyección en el futuro permite pensar de qué manera los adultos y los agentes de salud pueden favorecer el desarrollo de una visión del sí mismo en el futuro que sea significativa para el bienestar, la motivación y autorregulación del comportamiento actual y la exploración y formación de la identidad.

\section{Conclusiones}

Los adolescentes de la Ciudad de Buenos Aires (Argentina) proyectan su visión de sí mismos en el futuro en cinco grandes áreas: sus características personales o rasgos de personalidad; sus ocupaciones y su estilo de vida; su apariencia y aptitudes físicas; sus relaciones interpersonales; y su satisfacción general, aspiraciones y logros. Dentro de los dominios incluidos en estas áreas, un contenido idiosincrático de los adolescentes de Buenos Aires es el de las metas: el valor de tener metas y del compromiso con ellas. Asimismo, algunos adolescentes logran una articulación e integración entre sus PSs, lo cual implica una visión más compleja de ellos mismos a futuro. Los PSs más prevalentes son los referidos a la profesión y ocupación, y a los estudios e intereses académicos. El dominio del trabajo toma prevalencia entre los PSs temidos. Si bien los distintos tipos de PSs se expresan en las mismas áreas, hay algunas diferencias. En cuanto a la diversidad, la mayoría de los adolescentes generó PSs en una o dos áreas, siendo el máximo cinco áreas. $\mathrm{El}$ bajo porcentaje de adolescentes que pudieron generar PSs complejos y en más de una o dos áreas, así como la alta prevalencia de PSs en el ámbito académico/ocupacional lleva a considerar la necesidad de alentarlos a explorar una mayor diversidad de posibles sí mismos, a considerar otras áreas en las que proyectarse y a profundizar en las características de los PSs que se plantean a futuro, articulando diferentes dominios entre sí.

\section{Lista de referencias}

Aloise-Young, P. A., Hennigan, K. M. \& Leong, C. W. (2001). Possible selves and negative health behaviors during early adolescence. The Journal of Early Adolescence, 21 (2), pp. 158-181. http:// doi.org/10.1177/0272431601021002002

Bak, W. (2015). Possible selves: Implications for psychotherapy. International Journal of Mental Health and Addiction, 13 (5), pp. 650-658. http://doi.org/10.1007/ s11469-015-9553-2

Barber, B., Abbott, B., Blomfield, C. \& Eccles, J. (2009). Secrets of their success. Activity participation and positive youth development. In R. Gilman, E. S. Huebner \& M. J. Furlong (eds.) Handbook of Positive Psychology in Schools. New York: Taylor \& Francis.

Brewer, K. B., Gearing, R. E., Schwalbe, C. S. \& Ibrahim, R. W. (2013). Possible Selves and Risk Behavior Among Adolescents in Conflict with the Law in the Hashemite Kingdom of Jordan. In The Society for Social Work and Research 2013 Annual Conference. Retrieved from: https://sswr. confex.com/sswr/2013/webprogram/ Paper18730.html

Bronk, K. C., Hill, P. L., Lapsley, D. K., Talib, T. L. \& Finch, H. (2009). Purpose, hope, and life satisfaction in three age groups. The Journal of Positive Psychology, 4 (6), pp.500-510. http://doi. org/10.1080/17439760903271439

Cadely, H. S. E., Pittman, J. F., Kerpelman, J. L. \& Adler-Baeder, F. (2011). The role of identity styles and academic possible selves on academic outcomes for high 
school students. Identity: An International Journal of Theory and Research, 11 (4), pp. 267-288. http://doi.org/10.1080/1528 3488.2011 .613580

Destin, M.\& Oyserman, D. (2010). Incentivizing education: Seeing schoolwork as an investment, not a chore. Journal of Experimental Social Psychology, 46 (5), pp. 846-849. http://doi.org/10.1016/j. jesp.2010.04.004.Incentivizing

Erikson, E. H. (1968). Identidad, juventud y crisis. Buenos Aires: Paidós.

Erikson, M. G. (2007). The meaning of the future: Toward a more specific definition of possible selves. Review of General Psychology, 11 (4), pp. 348-358. http:// doi.org/10.1037/1089-2680.11.4.348

Harter, S. \& Bukowski, W. M. (2012). The construction of the self: Developmental and sociocultural foundations. New York: The Guilford Press.

Hernández-Sampieri, R., FernándezCollado, C. \& Baptista-Lucio, P. (2010). Metodología de la investigación [Research methodology]. México, D. F.: Mc Graw Hill.

Knox, M., Funk, J., Elliot, R. \& Bush, E. G. (1998). Adolescents' possible selves and their relationship to global selfesteem. Sex Roles, 39, pp. 61-80. Retrieved from: http://link.springer.com/ article/10.1023/A:1018877716225

Lee, C. K., Corte, C., Stein, K. F., Park, C. G., Finnegan, L. \& Mccreary, L. L. (2015). Prospective effects of possible selves on alcohol consumption in adolescents. Research in Nursing and Health, 38 (1), pp. 71-81. http://doi.org/10.1002/ nur. 21641

Malin, H., Reilly, T. S., Quinn, B. \& Moran, S. (2014). Adolescent purpose development: Exploring empathy, discovering roles, shifting priorities, and creating pathways. Journal of Research on Adolescence, 24 (1), pp. 186-199. http://doi.org/10.1111/ jora. 12051

Markus, H. \& Nurius, P. (1986). Possible Selves. American Psychologist, 41 (9), pp. 954-969. http://doi.org/10.1037/0003066X.41.9.954
Marshall, S. K., Young, R. A., Domene, J. F. \& Zaidman-Zait, A. (2008). Adolescent possible selves as jointly constructed in parent-adolescent career conversations and related activities. Identity: $A n$ International Journal of Theory and Research, 8, pp. 185-204. http://doi. org/10.1080/15283480802181727

McClelland, C. (2011). An examination of the present and future selves of adolescents: Investigating possible selves and selfesteem in relation to gender and attachment. The Plymouth Student Scientist, 4 (2), pp. 104-130. Retrieved from:

http://studentjournals.plymouth.ac.uk/ index.php/pss/article/viewArticle/179

Molina, M. F. (2014). Cuestionario de los posibles sí mismos. Material Inédito.

Molina, M. F., Gimenez, M., Raimundi, M. J., Leibovich, N. B. \& Schmidt, V. (2015). ¿Cómo perciben los adolescentes de la Ciudad de Buenos Aires su futuro? Una mirada desde sus actores. Manuscrito Presentado Para Su Publicación.

Núñez, L. \& Romero, C. (2003). Pensar la educación. Conceptos y opciones fundamentales. Madrid: Pirámide.

Oyserman, D. (2001). Self-concept and identity. In A. Tesser \& N. Schwarz (eds.) Blackwell handbook of social psychology: Intraindividual processes, (pp. 499-517). Massachusetts: Blackwell.

Oyserman, D. (2004). Possible selves citations, measure, and coding instructions. Retrieved from: http://www.sitemaker. umich.edu/ culture.self/files/possible selves_measure.doc

Oyserman, D., Bybee, D. \& Terry, K. (2006). Possible selves and academic outcomes: How and when possible selves impel action. Journal of Personality and Social Psychology, 91 (1), pp. 188-204. http:// doi.org/10.1037/0022-3514.91.1.188

Oyserman, D., Destin, M. \& Novin, S. (2015). The context-sensitive future self: Possible selves motivate in context, not otherwise. Self and Identity, 14 (2), pp. 173-188. http://doi.org/http://dx.doi.org/10.1080/1 5298868.2014 .965733 
Oyserman, D. \& Fryberg, S. (2006). The possible selves of diverse adolescents: Content and function across gender, race and national origin. In C. Dunkel \& J. Kerpelman (eds.) Possible selves: Theory, research, and applications, (pp. 1-23). New York: Nova Science Publishers.

Oyserman, D. \& James, L. (2009). Possible selves: From content to process. In K. D. Markman, W. M. Klein \& J. A. Suhr (eds.) Handbook of imagination and mental simulation, (pp. 373-394). New York: Taylor \& Francis.

Oyserman, D. \& James, L. (2011). Possible identities. In S. J. Schwartz, K. Luyckx \& V. L. Vignoles (eds.) Handbook of Identity Theory and Research, (pp. 117-145). New Jersey: Springer Link. http://doi. org/10.1007/978-1-4419-7988-9

Oyserman, D. \& Markus, H. (1990). Possible selves and delinquency. Journal of Personality and Social Psychology, 59 (1), pp. 112-125. Retrieved from: http://www. ncbi.nlm.nih.gov/pubmed/2213484

Pierce, J., Schmidt, C. \& Stoddard, S. A. (2015). The role of feared possible selves in the relationship between peer influence and delinquency. Journal of Adolescence, 38, pp. 17-26. http://doi.org/10.1016/j. adolescence.2014.10.009

Prince, D. (2014). What about place? Considering the role of physical environment on youth imagining of future possible selves. Journal of Youth Studies, 17 (6), pp. 697-716. http://doi.org/http:// dx.doi.org/10.1080/13676261.2013.83659 1 What

Raimundi, M. J., Molina, M. F., Gimenez, M. \& Minichiello, C. (2014). ¿Qué es un desafío? Estudio cualitativo de su significado subjetivo en adolescentes de Buenos Aires. Revista Latinoamericana de Ciencias Sociales, Niñez y Juventud, 12 (2), pp. 521-534. http://doi.org/10.11600/ $1692715 x .1221110414$

Shepard, B. \& Marshall, A. (1999). Possible selves mapping: Life-career exploration with young adolescents. Canadian Journal of Counselling, 33 (1), pp. 37-54. Retrieved from: http://eric.ed.gov/?id=EJ587281
Sica, L. S. (2009). Adolescents in different contexts: The exploration of identity through possible selves. Cognition, Brain, Behavior. An Interdisciplinary Journal, XIII (3), pp. 221-252. Retrieved from: psycnet.apa.org/psycinfo/2009-21493-001

Strauss, A. \& Corbin, J. (1990). Basics of qualitative research: Grounded theory procedures and techniques. London: Sage Publications.

Velandia-Morales, A. (2008). Investigación cualitativa y psicología del consumidor: alternativas de aplicación. Avances en Psicología Latinoamericana, 26 (2), pp. 290-303.

Watson, D. (2002). Positive affectivity. The disposition to experience plesurable emotional states. In C. R. Snyder \& S. Lopez (eds.) Handbook of Positive Psychology. New York: Oxford University Press.

Zhu, S. \& Tse, S. (2015). Possible selves, strategies and perceived likelihood among adolescents in Hong Kong: Desire and concern. International Journal of Adolescence and Youth. Advance on Line Publication. http://doi.org/10.1080/02673 843.2015.1031683 